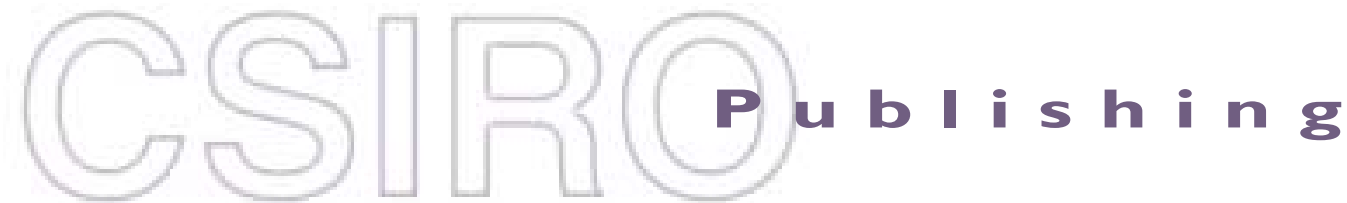

\section{Publications of the Astronomical Society of Australia}

Volume 19, 2002

(C) Astronomical Society of Australia 2002

An international journal of astronomy and astrophysics

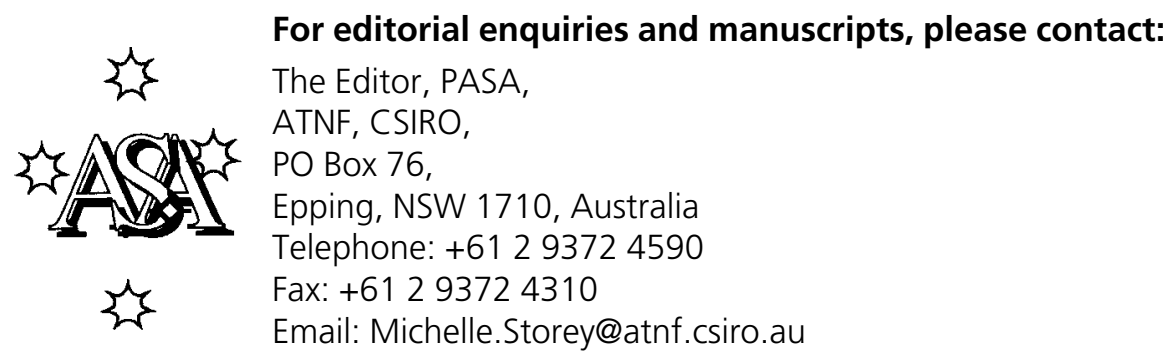

For general enquiries and subscriptions, please contact: CSIRO Publishing PO Box 1139 (150 Oxford St)

Collingwood, Vic. 3066, Australia

Telephone: +6139662 7666

Fax: +61 396627555

Email: publishing.pasa@csiro.au

C S I RO

PUBLISHING Published by CSIRO Publishing

for the Astronomical Society of Australia

www.publish.csiro.au/journals/pasa 


\title{
Annual Modulation in the Variability Properties of the IDV Source 0917+624?
}

\author{
L. Fuhrmann ${ }^{1}$, T. P. Krichbaum ${ }^{1}$, G. Cimò ${ }^{1}$, T. Beckert ${ }^{1}$, A. Kraus ${ }^{1}$, \\ A. Witzel ${ }^{1}$, J. A. Zensus ${ }^{1}$, S. J. Qian ${ }^{1,2}$ and B. J. Rickett ${ }^{3}$ \\ ${ }^{1}$ Max-Planck-Institut für Radioastronomie, Bonn \\ ${ }^{2}$ Beijing Astronomical Observatory, China \\ ${ }^{3}$ University of California, San Diego
}

Received 2001 August 30, accepted 2001 November 27

\begin{abstract}
There is new evidence which identifies seasonal changes of the variability time scale in intraday variable (IDV) sources with refractive interstellar scintillation effects. Such a RISS model takes the annual change of the Earth's velocity relative to the scattering medium into account. In September 1998 we found a remarkable prolongation of the variability time scale in the IDV source $0917+624$ with only small variations in flux density during a period of 5 days. This was explained as a seasonal effect, in which the velocity vector of the Earth and the interstellar medium nearly cancelled. In order to further investigate the applicability of the model for $0917+624$, we performed an Effelsberg $6 \mathrm{~cm}$-flux monitoring program over the course of one year. Since September 2000, the source appears to be remarkably inactive and yet (May 2001) no return to its normal, faster and stronger variability pattern is observed. Here, our observational results and a possible explanation for the current quiescence are presented.
\end{abstract}

Keywords: quasars: individual $(0917+62)$ — radio continuum: ISM — scattering

\section{Introduction}

The quasar $0917+624$ was one of the sources in the original IDV sample in 1985, which showed variability on time scales shorter than one day (Heeschen et al. 1987). Since then, the source was regularly monitored over the last 15 years and generally was found to be strongly variable with amplitude variations on a 10-15\% level and on time scales in the range of 0.8-1.6 days. Furthermore, faster variability is detected in the polarised flux and the polarisation angle. In polarisation, larger amplitude variations (up to a factor of 2) are common, and once a $180^{\circ}$ swing in the polarisation angle was observed (Quirrenbach et al. 1989). In September 1998, however, Kraus et al. (1999) detected a remarkable change in the variability properties: the rapid variability stopped and only a slow increase of $7 \%$ in flux over the 5-day observing session has been observed (Figure 1). Observations in February 1999 then showed $0917+624$ to be variable again on a time

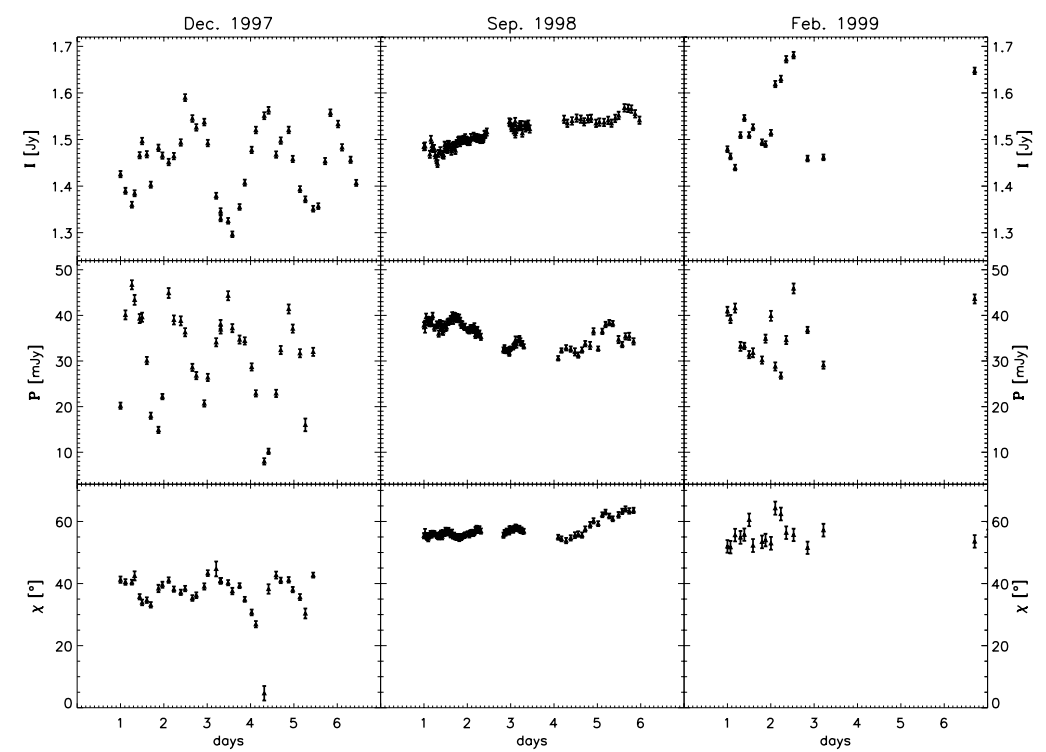

Figure 1 Observations of 0917+624 by Kraus et al. (1999) between December 1997 and February 1999. From top to bottom: total flux density I, polarised flux density P, and polarisation angle $\chi$ against time. Note the remarkable 'slow down' in September 1998 and the return to fast variability in February 1999. 
Projected velocity of the earth

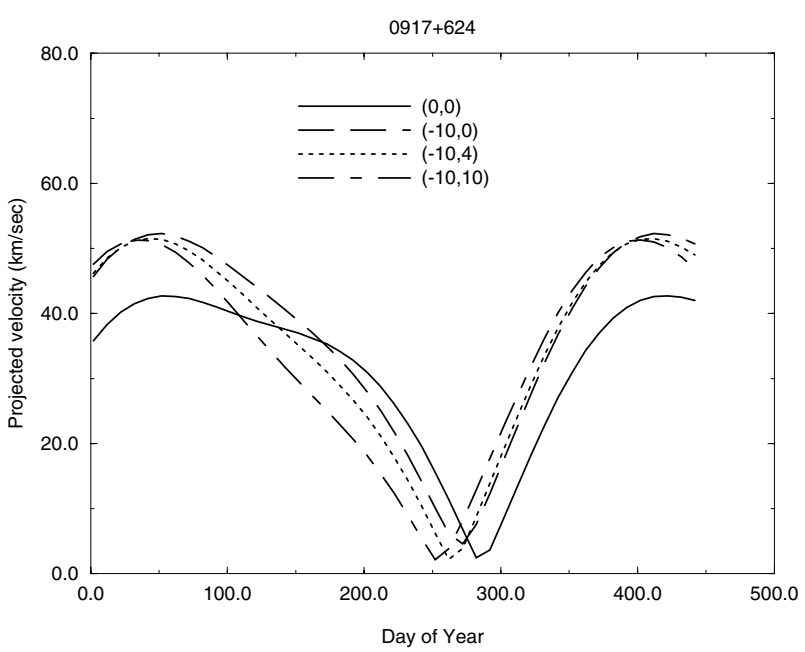

Annual Modulation of Time Scale

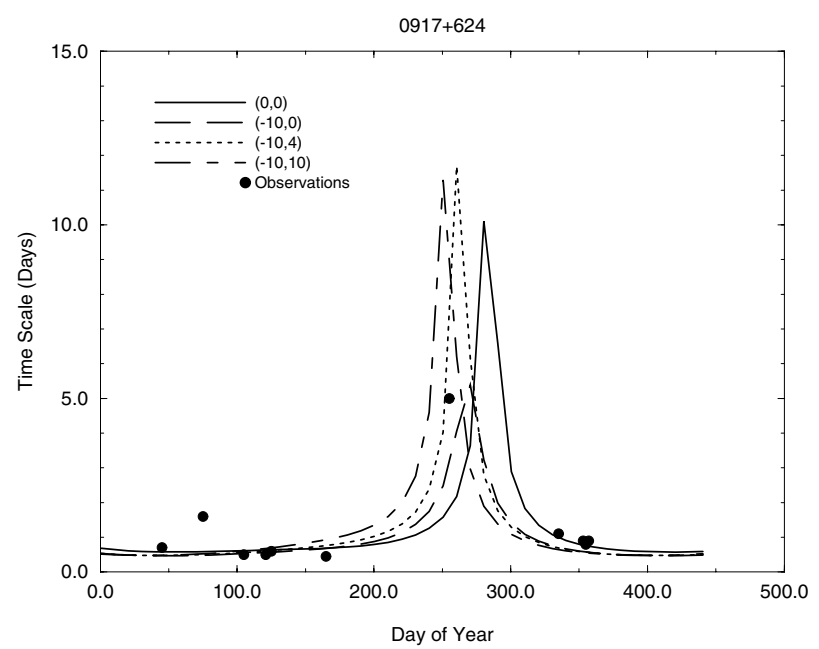

Figure 2 Left: the velocity of the Earth relative to the LSR projected perpendicular to the direction of $0917+624$ versus day of the year for four different velocities of the screen. Note the strong decrease near day 285. Right: resulting 'slow-down-peak' in the predictions of the variability time scale. We overplot the predicted and the observed time scales and find a good agreement (see text).

scale of 1.3 days. Kraus et al. (1999) interpreted the slowdown in September as probably due to the ejection of a new jet component. This temporarily leads to a source size larger than the scattering size (set by the ISM) and a reduction of the observed refractive interstellar scintillation (quenched RISS) (Rickett et al. 1995). An alternative possibility to explain the behaviour of $0917+624$ within an ISS model was given by Rickett et al. (2001) (see also Jauncey \& Macquart 2001). They suggested that the observed change in the variability time scale depends on the time of the year and thus reflects the orbital motion of the Earth relative to the ISM. Hence, using an ISS model for $0917+624$ as suggested by Rickett et al. (1995) and taking the Earth's orbital motion around the Sun with respect to the scattering medium into account leads to a predicted slow-down in September and could explain the prolongation in September 1998. Since we have only one data point during this highly interesting time period, we performed a dedicated flux density monitoring program for $0917+624$ over the course of one year, in order to test the annual modulation of IDV in this source (and two others).

\section{Annual Modulations in IDV}

Recent evidence for a seasonal dependence of the variability time scale comes from the highly variable quasar J1819+3845 (Dennett-Thorpe \& de Bruyn 2000). Westerbork observations during a period of 12 months indicate a variation of the IDV time scale by a factor of $\sim 10$ and a clear 'slow-down-peak' near day 280. In the following year the prolongation could be reproduced nicely (A. G. de Bruyn 2001, private communication). However, in addition to the Earth's motion with respect to the local standard of rest (LSR), a medium velocity of $\sim 25 \mathrm{~km} \mathrm{~s}^{-1}$ is required.
In the standard ISS model, the observer moves through a spatial scintillation pattern caused by a screen of scattering material at a distance $\mathrm{D}$. The variability time scale is then determined by two quantities: the spatial scale of the pattern, and the observer's motion projected onto the scintillation pattern. In order to explain the annual variation of the IDV time scale in terms of ISS, the relative motion of the Earth with respect to the scattering medium is an important parameter and has to be taken into account. This relative motion is composed of three different velocities: i) the Earth's orbital motion around the Sun; ii) the Sun's motion towards the solar apex; iii) the velocity of the medium with respect to the LSR. In Figure 2 the velocity of the Earth relative to the LSR projected perpendicular to the direction of $0917+624$ is displayed versus day of the year. Near day number 285 the velocity of the Earth's orbital motion nearly cancels the projected velocity of the Sun relative to the LSR. This effect leads to a seasonal dependence in the variability time scale: while the predicted time scale should remain nearly constant over a large fraction of the year, a substantial prolongation in September and October (days 250-330) should occur. In Figure 2 we overplot the predicted and observed time scales and we find a general agreement between the observations and the predictions. The data point in September from Kraus et al. (1999) lies in the period of the predicted increase. The other data points come from our observations performed during 1989-2000.

\section{Observations and Data Reduction}

In order to investigate the predictions of the above model for $0917+624$ in more detail, we started a monitoring program at $6 \mathrm{~cm}$ wavelength over the last year. The observations were performed from September 2000 to May 2001 at the $100 \mathrm{~m}$ telescope of the MPIfR in Effelsberg. 


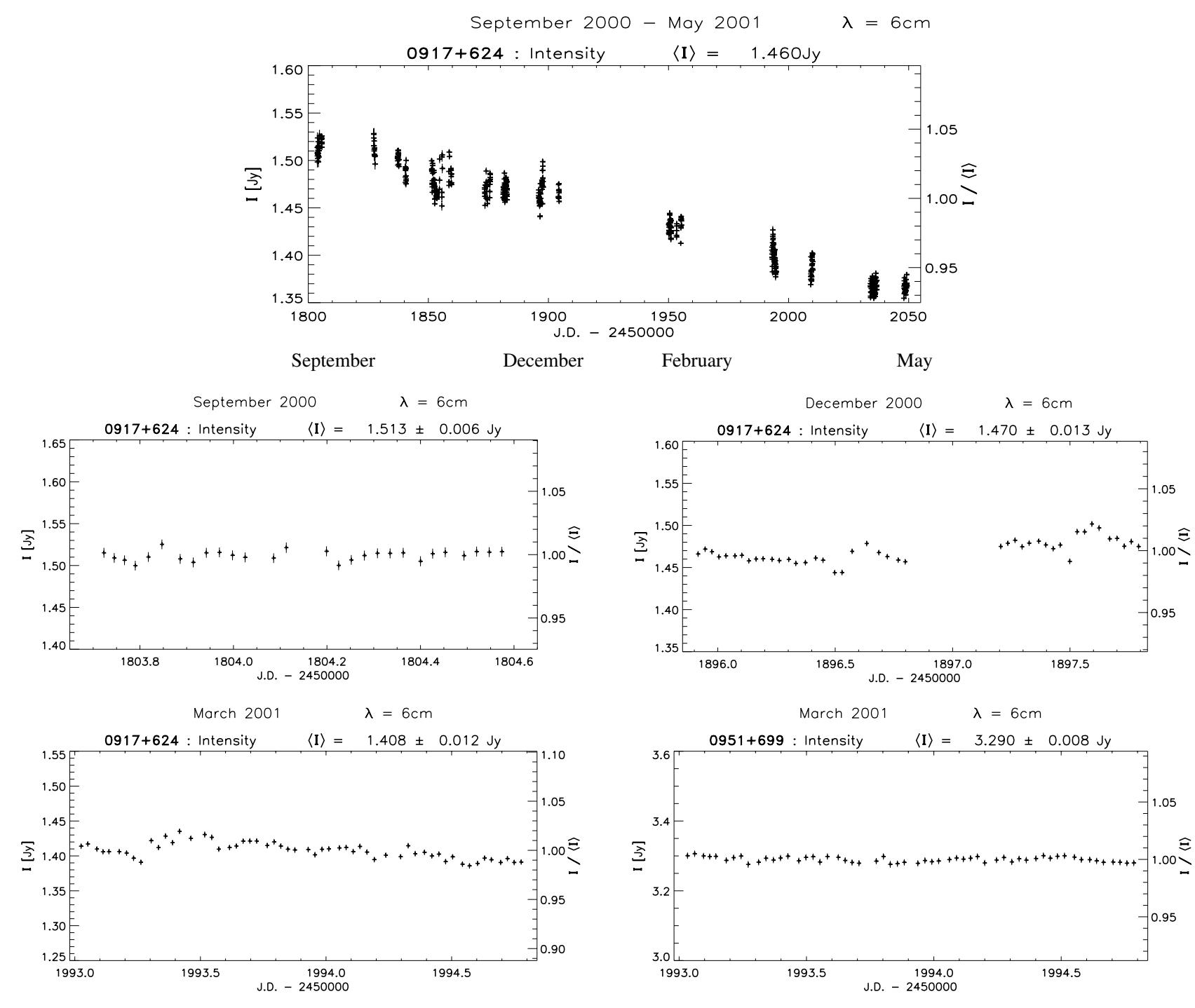

Figure 3 Summary of our observations between September 2000 and May 2001. At the top, the long term light curve of 0917+624 with a trend of $\sim 10 \%$ decrease is displayed. In addition, three individual light curves from September $2000(m=0.4 \%)$, December $2000(m=0.9 \%)$, and March $2001(m=0.8 \%)$ are plotted. For comparison, the lower right panel shows one of our secondary calibrators in March $2001(m=0.2 \%)$.

During this time 22 single observing sessions with a duration of a few hours up to three days were performed. In this experiment $0917+624$ was one source within a small sample of IDV sources and has been observed with a dense duty cycle of about two measurements every $1.5 \mathrm{hr}$. In addition, two non-IDV sources, 0951+699 and 0836+73, were included as secondary calibrators. We observed standard flux density calibrators like 3C286, 3C295, 3C48, and NGC7027 frequently. Since all measured sources are point-like and sufficiently strong at $6 \mathrm{~cm}(\geq 0.5 \mathrm{Jy})$ we were able to measure the flux densities with cross-scans in azimuth and elevation (e.g. Quirrenbach et al. 1992; Kraus 1997). We used the standard IDV data reduction procedure of the Effelsberg telescope (for details see Quirrenbach et al. 1992 and Kraus 1997). Our secondary calibrators were observed with similar duty cycles as the program sources to allow for a systematic elevation- and timedependent correction of the measured flux densities. The resulting flux density errors are composed of the statistical errors from the reduction process (Gaussian fits of averaged sub-scans) and a contribution from the systematics as seen in our secondary calibrators (weather). Our final errors lie in the range of $\leq 0.5-1 \%$.

Since a polarimeter is integrated in the $6 \mathrm{~cm}$ receiver, we also obtained full polarisation information. The reduction and analysis of the polarisation data is still in progress and will be discussed elsewhere (L. Fuhrmann et al., in preparation).

\section{Results}

In Figure 3 we summarise our results. Since September 2000, all 22 individual light curves show a very inactive source with only few per cent amplitude variations. At the top of Figure 3 the long term light curve of $0917+624$ over the full monitoring period of nine months is displayed. During this time interval, we detected a long term trend with a monotonic $\sim 10 \%$ decrease in total 

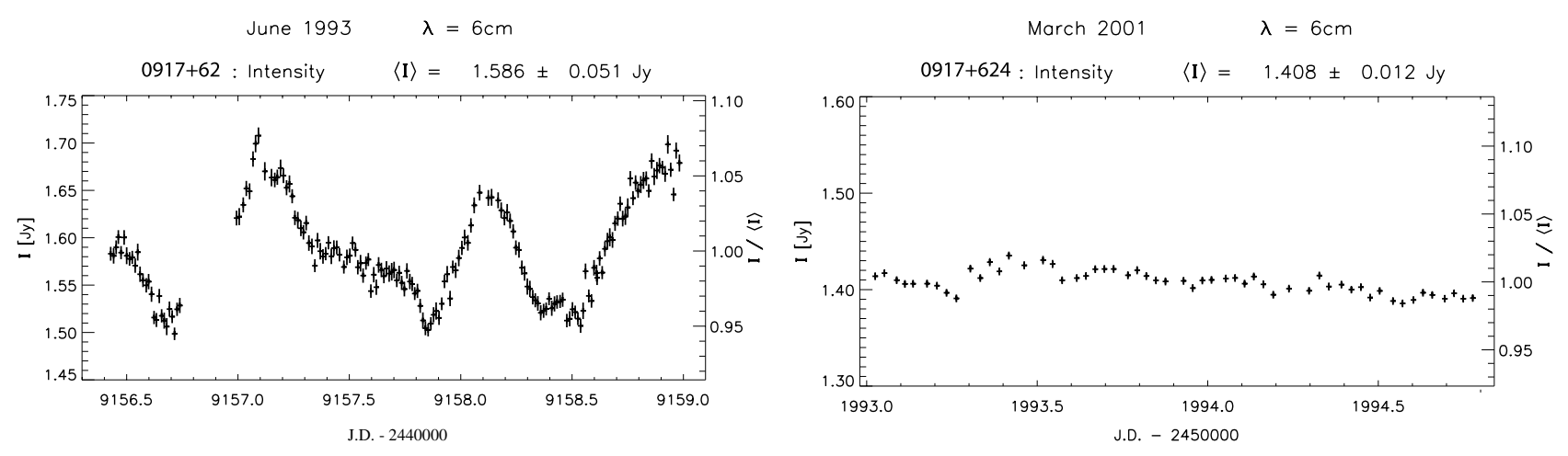

Figure 4 Comparison of two periods with different variability pattern. The left curve shows 0917+624 in June 1993 with its normal, strong variability pattern $(m=3.3 \%)$. On the right a recent light curve from March 2001 (plotted on the same scale) is displayed $(m=0.8 \%)$. Note the low variations compared to the past.

flux since October 2000. In addition, Figure 3 shows three individual light curves measured in September 2000, December 2000, and March 2001. For comparison, the March 2000 light curve of one of our secondary calibrators $(0951+699)$ is displayed. All curves of $0917+699$ show only very low amplitude variations of about $2-3 \%$ ('ripples') on very short time scales of $\sim 0.3-0.4$ days. Since September, no significant IDV is seen in $0917+624$ and the strong, pronounced IDV seen at earlier times is completely missing.

\section{Discussion}

It can be seen from Figure 3 that $0917+624$ passed through a dramatic change of its variability properties when compared to the past. The change can clearly be seen by plotting a light curve of our new campaign on the same scale with data from earlier observations. This is realised in Figure 4: while in June 1993 0917+624 showed its normal, strong variability pattern with $\sim 15 \%$ amplitude variations on a time scale of $\sim 0.5$ days, the variations in March 2001 appear dramatically low. In order to compare the strength of the variability, we determined the modulation index $m=100 \times \sigma_{S} /\langle S\rangle$ where $\sigma_{S}$ is the rms flux density variation. It can be seen from Figure 5 that during our recent monitoring $m$ has always been low: $m \leq 0.9$. In the most extreme case, this is a factor $\sim 7$ lower than the variations observed in the past. In order to explain the behaviour of $0917+624$ in the context of RISS and the annual modulation effect, however, we see three main problems: i) Other IDV sources in the same region of the sky (e.g. 0716+71, 0954+65) do not show the expected seasonal dependence of the variability time scale. ii) The orbital motion of the Earth should affect the time scale, but not the modulation index $m$ (Rickett et al. 2001), which also appears to be variable (Figure 5). iii) Our monitoring program presented here has not yet revealed a restart of the strong and pronounced variability in $0917+624$ since September 2000. This contradicts the model predictions of Rickett et al. (2001), which claimed recovery to the 'normal' variability already at the end of last year (see Figure 2).
We suggest two possible scenarios in order to explain the present anomalous behaviour of 0917+624. Firstly, the interstellar medium is far more complex than presently thought and a change in the scattering properties of the screen might explain the change of the variability mode in $0917+624$. If the spatial scale of the scintillation pattern decreased, then the source size could quench the ISS. For weak ISS or RISS this would imply either a decrease in the strength of turbulence in small, localised regions of the ISM or an increase of the distance to such a region. In turn this would require a very inhomogeneous ISM, with moving 'clouds' or 'layers' at different distances. The inhomogeneities then have to occur on angular scales of less than the angular separation from $0917+62$ to other sources $\left(\leq 5-10^{\circ}\right)$, the latter still showing pronounced IDV. In this context, the present situation of nearly no variations in $0917+624$ could be explained by either a 'hole' in the screen, or a moving foreground layer which was present during the last 15 years and has suddenly disappeared within the last year.

Secondly, an alternative interpretation could be based on intrinsic variations in the VLBI structure of the source between 1998 and 2000. The dominant components, which in the past were responsible for RISS, either disappeared or increased their intrinsic size, hence producing quenched scattering. Recent evidence for such an effect is coming from VLBI observations at $15 \mathrm{GHz}$ (see Krichbaum et al. 2002). Multi-epoch observations during 1999 and 2000 reveal structural expansion in the central region with 5-6c. Extrapolating the motion of one jet component backwards in time leads to a time of ejection shortly before the date when $0917+624$ ceased varying for the first time (September 1998, Kraus et al. 1999). It is therefore very tempting to assume that the present quiescence in the variability of $0917+624$ once more is caused by blending effects of the inner, scintillating region by another new component. This component probably was ejected during the first half of the year 2000 and should become visible in future VLBI observations.

We conclude that the annual modulation effect in $0917+624$ may at present not be visible due to structural changes in the central region, and hence only strongly 

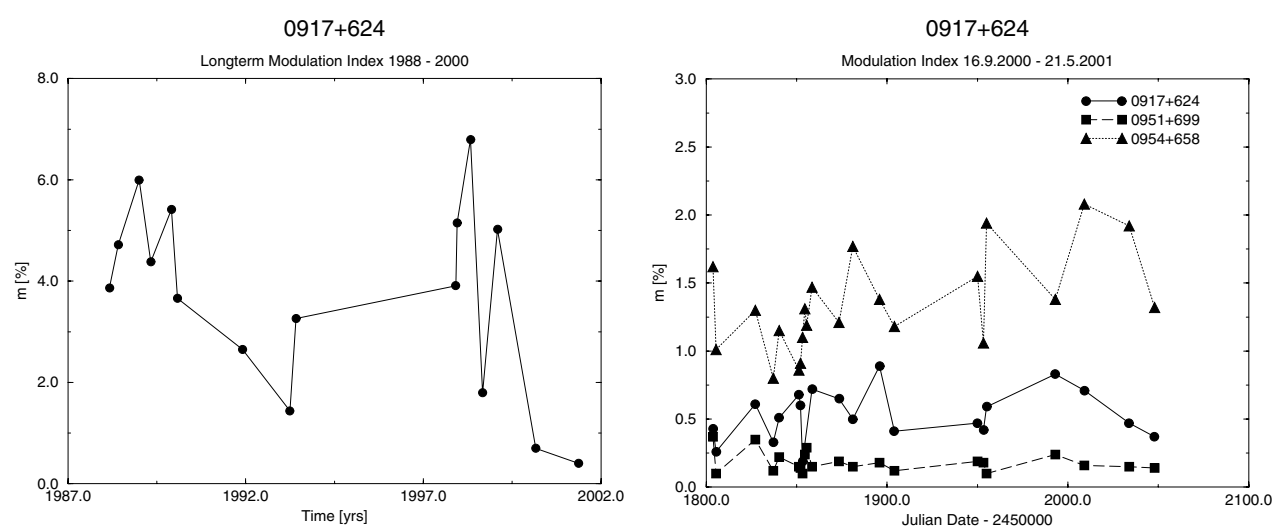

Figure 5 Left: long term modulation index distribution between 1988 and 2000 representing the strong variability of $0917+624$ up to year 2000. Right: modulation index during our monitoring campaign 2000/2001. In addition, the IDV source $0954+658$ and the secondary calibrator $0951+699$ are displayed.

quenched scattering could be observed during the last nine months. In order to detect a seasonal dependence of the variability time scale, less complex and more pointlike sources (e.g. J1819+3845) would provide a better opportunity to investigate the annual modulation model.

\section{References}

Dennett-Thorpe, J., \& de Bruyn, A. G. 2000, ApJ, 529, L65

Heeschen, D. S., Krichbaum, T. P., Schalinski, C. J., \& Witzel, A. 1987, AJ, 94, 1493
Jauncey, D. L., \& Macquart, J.-P. 2001, A\&A, 370, L9

Kraus, A., Witzel, A., Krichbaum, T. P., Lobanov, A. P., Peng, B., \& Roset, E. 1999, A\&A, 352, L107

Kraus, A. 1997, PhD Thesis, University of Bonn, Germany

Krichbaum, T. P., Kraus, A., Fuhrmann, L., Cimò, G., \& Witzel, A. 2002, PASA, 19, 14

Quirrenbach, A., Witzel, A., Qian, S. J., Krichbaum, T. P., Hummel, C. A., \& Alberdi, A. 1989, A\&A, 226, L1

Quirrenbach, A., et al. 1992, A\&A, 258, 279

Rickett, B. J., Witzel, A., Kraus, A., Krichbaum, T. P., \& Qian, S. J. 2001, ApJ, 550, L11

Rickett, B. J., Quirrenbach, A., Wegner, R., Krichbaum, T. P., \& Witzel, A. 1995, A\&A, 293, L479 